\title{
Presentation of the hemisphere method
}

\author{
P. Vueghs \& P. Beckers \\ Aerospace and Mechanical Engineering Department, \\ University of Liege, Belgium
}

\begin{abstract}
In the field of image synthesis or thermal radiation, the challenge is to calculate the radiative exchanges between the surfaces of the model. To quantify the interactions between the surfaces, we define a number called view factor, noted $F_{i j}$. The view factor represents the fraction of the diffusely emitted power from a surface $A_{i}$ which reaches a surface $A_{j}$. The calculation of the view factors is a very difficult problem. A method has been designed in image synthesis and also applied to thermal problems. This method, called hemi cube, uses a cube as a screen onto which all the scene is projected. Here, we present another method that we have named hemisphere, where the surface of projection is the unit sphere surrounded by the hemi cube. This projection, based on the Nusselt's Analogy, is simpler and more natural than the hemi cube's projection. We show in this paper that the hemisphere method is faster and more efficient than the hemi cube. In this paper, we present a tessellation of the hemisphere into cells characterized by an equal elementary view factor. This allows us to avoid over sampling and to save computation time. We show that this method is faster and more efficient than the classic hemi cube. We present also some results that illustrate the efficiency of the hemisphere method.

Keywords: view factor, hemisphere, projection, spherical coordinates, thermal radiation, Nusselt's Analogy, ray casting.
\end{abstract}

\section{Definition of the view factors}

In the field of image synthesis, it is necessary to calculate the inter reflection of light between all the surfaces that compose the scene [9]. In the same way, in thermic, we have to evaluate all the heat transfers between all the surfaces of the model. 
The exchanges of energy between two surfaces depend on the geometrical configuration of these surfaces. Mathematically, this geometrical dependency is expressed by a function called view factor [2]. The view factor expresses that the exchange of energy between two surfaces depends on their sizes, their relative orientations and the distance between them. The view factor does not depend on the radiative properties of the surfaces, such as absorbance and emittance.

By definition, the view factor between two surfaces $A_{i}$ and $A_{j}$ is the fraction of the energy emitted diffusely by the surface $A_{i}$ which reaches the surface $A_{j}$. We can note here the ambiguity of this definition based on the notions of emitted and absorbed energy although only the geometrical parameters appear in the definition, the surface properties do not appear.

According to [14], if we assume that two surfaces $A_{i}$ and $A_{j}$ are fully visible to each other, the diffuse view factor between these surfaces is given by

$$
F_{A_{i}-A_{j}}=\frac{1}{A_{i}} \int_{A_{i}} \int_{A_{j}} \frac{\cos \theta_{i} \cos \theta_{j}}{\pi r_{i j}^{2}} d A_{i} d A_{j}
$$

where $\theta$ is the angle between the normal vector at the surface and the vector $\vec{r}_{i j}$ which joins points on the two surfaces.

This expression has no analytical solution, except in the case of particular geometrical configurations $[3,6,7]$. It implies a numerical resolution of the integration involved in the computation of the view factors. Several techniques have been designed. If we consider the view factor between a point on the surface $i$, noted $d A_{i}$, and the surface $A_{j}$, eqn (1) can be simplified. It is the limit of the usual view factor when the area $A_{i}$ goes to zero. This new view factor is called pointwise view factor and is the inner integral of eqn (1)

$$
F_{d A_{i}-A_{j}}=\int_{A_{j}} \frac{\cos \theta_{i} \cos \theta_{j}}{\pi r_{i j}^{2}} d A_{j}
$$

We can evaluate this expression by different analytical methods. The most usual in image synthesis is called hemi cube [13].

The method presented in this article is mainly based on the hemi cube method. The principle is the same. It is also linked to the idea presented by F. Sillion to decompose the projection surface into cells of equal view factors [4]. F. Sillion proposed to project all the environment onto a single plane which takes the place of the top face of the hemi cube. He introduced a quadrangular mesh of this plane. The cells of this mesh are characterized by approximately equal elementary view factors. This idea has also been developed by Vivo et al. [11] and Lluch et al. [16]. They used the polar coordinates to obtain cells of equal view factors in the single plane. With this method, they conserve the axial symmetry of the formula of the view factor. The main disadvantage of the single plane methods is that the portion of the environment situated near the horizon cannot be taken into account. However, if the size of the plane is sufficient, we can reduce the neglected portion to a given threshold. 
Here, we choose to project the scene onto the hemisphere because it is easier to mesh this surface than a plane of a cube, if we want to obtain cells of equal view factors. We obtain a very regular mesh which respects the axial symmetry of the view factor. We can also guarantee that the entire scene is taken into account in this method, unlike in the single plane methods.

\section{The hemi cube}

The hemi cube was designed and presented in 1985 by Donald P. Greenberg and Michael F. Cohen [13]. It was first used in image synthesis and after to solve thermal analysis [10].

This method consists of drawing a cube centered around the origin. The $z$ axis coincides with the normal vector $\vec{n}_{i}$. The construction is represented in figure 1 according to references $[8,13]$.

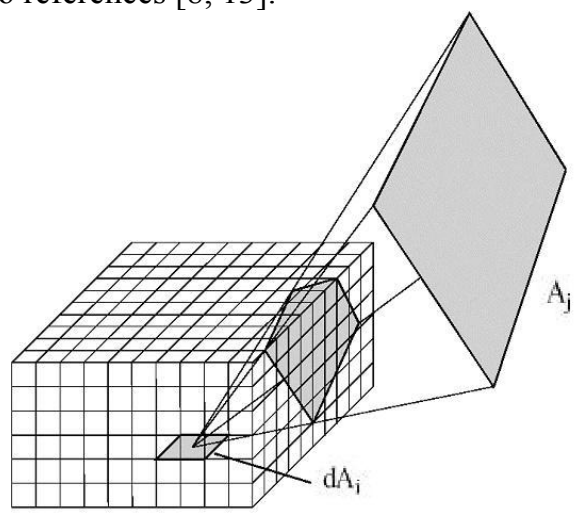

Figure 1: Principle of the hemi cube.

The cube is discretized into a number of cells. Each cell is associated with an element of view factor, called delta view factor. These elements are contributions to the pointwise view factor. Each value depends on the position of the cell on the hemi cube. The cells near the $z$ axis are associated with the highest values and the cells located near the edges are characterized by lower values. This remark explains why we have over sampling.

This method presents drawbacks:

- an irregular sampling of the space, which implies that some areas are over sampled to guarantee a minimal accuracy.

- the contributions to the pointwise view factor are not equal. We need to compute the value and to store it for each cell of the hemi cube.

Nevertheless, the main advantage of this technique is that we can easily take occlusions into account by performing a test based on the distance of the surfaces projected onto the hemi cube. If many surfaces are projected onto the same cell of the hemi cube, we only keep the nearest one, the others will not be visible from the origin. 


\section{Distribution of elements of equal view factors}

In this section, we will present the solution we chose to obtain a regular mesh of cells with equal view factors. First, we will call back the definition of the view factor and the Nusselt's Analogy. After that, we will mesh the hemisphere in spherical coordinates, in colatitude $\theta$ and in longitude $\varphi$.

\subsection{Definition of the view factor and Nusselt's Analogy}

According to Puech and Sillion [5], the view factor between a point $x_{i}$ on a surface $A_{i}$ and a surface $A_{j}$ (fully visible from $x_{i}$ ) is noted $F_{d A_{i}-A_{j}}$ and is given by eqn (2).

Note that to establish this equation, we assumed that patch $j$ is fully visible from $x_{i}$. In other formulations, the visibility function appears explicitly. This function is defined between two points, it is equal to 1 if the points are mutually visible and 0 otherwise. This function is discontinuous and increases the difficulty of the computation.

If we use the Nusselt's Analogy (cfr [15] cited by [5]), the pointwise view factor $F_{d A_{i}-A_{j}}$ can be considered as the result of two successive projections:

- a first projection onto the unit sphere centered on $x_{i}$. This step corresponds to the factor $\frac{\cos \theta_{j}}{r_{i j}^{2}}$ in the relation (2). The solid angle subtended by the surface $d A_{j}$ is given by $d \omega_{j}=\frac{\cos \theta_{j} d A_{j}}{r_{i j}^{2}}$.

- a second orthogonal projection down onto the plane of the surface $A_{i}$, which corresponds to the factor $\cos \theta_{\mathrm{i}}$.

Now we can transform the relation (2). We obtain the following expression

$$
F_{d A_{i}-A_{j}}=\int_{\Omega_{j}} \frac{\cos \theta_{i}}{\pi} d \omega_{j}
$$

where $\Omega_{\mathrm{j}}$ is the solid angle subtended by $A_{j}$.

\subsection{Mesh of the hemisphere}

Now, with the help of the Nusselt's Analogy, we will decompose the hemisphere into solid angles. Each solid angle will be characterized by the same view factor. The decomposition will be performed following the meridians and parallels, i.e. in terms of latitude and longitude. The total number of cells $N$ will be obtained by multiplying the numbers of subdivisions into the longitude and latitude directions, $n_{\text {lon }}$ and $n_{\text {lat }}$. 


\subsubsection{In terms of colatitude}

In figure 2, if we consider an elementary surface $d A_{i}$ and a spherical cap (of unit radius) defined between $\theta=0$ and $\theta=\theta_{\mathrm{S}}$, the view factor is calculated by

$$
F_{d A_{i}-A_{j}}=\frac{1}{\pi} \int_{0}^{2 \pi} \int_{0}^{\theta_{S}} \cos \theta \sin \theta d \theta d \varphi=\sin ^{2} \theta_{S}
$$

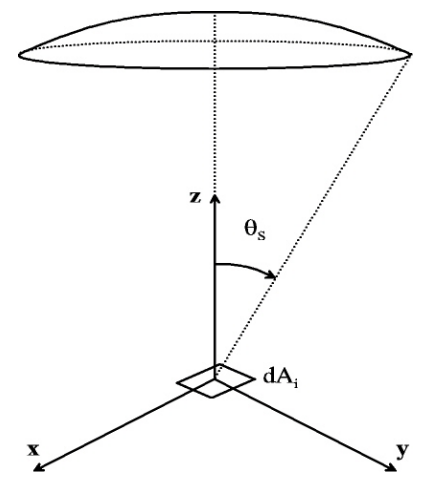

Figure 2: Spherical cap of angular aperture $\theta_{\mathrm{S}}$.

By extension, we can determine a sequence of values $\theta$ which correspond to spherical caps larger and larger. These caps delimit rings centered on the normal vector at the origin. The rings are characterized by a same value of the view factor with respect to the center of the emitting surface. If we fix the resolution in colatitude $n_{\text {lat }}$, we can subdivide the hemisphere in spherical rings with equal view factors.

\subsubsection{In terms of longitude}

After that, we can chose the discretization of the hemisphere in longitude simply by dividing the rings in a determined number $n_{\text {lon }}$ of sectors. All the sectors must have the same angular aperture.

\subsubsection{Resulting mesh}

So we have subdivided the hemisphere along the two spherical coordinates $\theta$ (colatitude) and $\varphi$ (longitude). The view factor associated to each cell is equal to $\frac{1}{n_{\text {lat }} n_{\text {lon }}}$. Figure 3 shows the hemisphere subdivided in longitude and latitude.

The cells are quadrangular, except the cells which have a vertex vertically above the center of the hemisphere and which are triangular. 


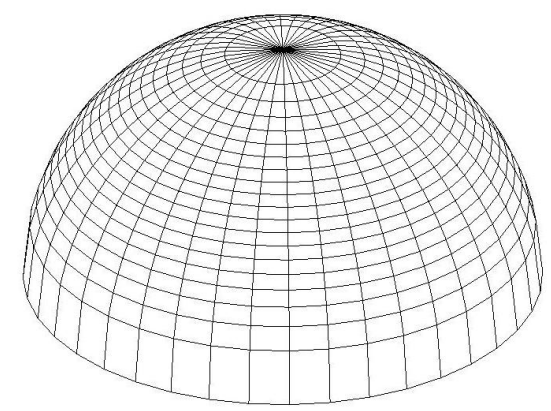

Figure 3: Wireframe view of the hemisphere.

\section{Calculation of the view factor}

The origin $d A_{i}$ is placed in a more or less complicated scene. The complexity of the scene is linked to the number of surfaces which compose the scene. The number of view factors to compute is equal to the number of surfaces in the scene.

After meshing the hemisphere, we cast a ray along each direction $\{\theta, \varphi\}$. For each ray, we first compute all the possible intersections with all the surfaces of the scene.

If no intersection is detected, this means that the model presents an aperture. For example, this is the case of a satellite which orbits around the Earth and evacuates the power dissipated by its equipments to the deep space at $3 \mathrm{~K}$. The contribution to the view factor is added to a node which does not belong to the model. This supplementary node represents the deep space and gathers all the energy fluxes emitted by the spacecraft.

When only one intersection is detected, the contribution to the view factor is assigned to the intersected surface. If several intersections are detected along the same direction, we store the distance of each intersection. At the end of the process, we only keep the nearest intersected surface.

This technique allows us to solve the visibility problem very easily and quickly.

\section{Error analysis}

The hemisphere method has been programmed and tested. The results are shown below.

Among the several tests we have performed, we chose to present the following one. For 2 perpendicular rectangles sharing a common edge, the aspect ratio of the rectangles is $D$ and $E$, with respect to the common edge. In this case, it is easy to obtain an analytical solution of the view factor between the two surfaces. In accordance to [6], the resulting formula is given below 


$$
\begin{gathered}
F_{A_{i}-A_{j}}=\frac{1}{\pi E}\left(E \arctan \left(\frac{1}{E}\right)+D \arctan \left(\frac{1}{D}\right)\right. \\
-\sqrt{D^{2}+E^{2}} \arctan \left(\frac{1}{\sqrt{D^{2}+E^{2}}}\right)+\frac{1}{4} \ln \left\{\frac{\left(1+E^{2}\right)\left(1+D^{2}\right)}{1+D^{2}+E^{2}}\right. \\
\left.\left.\left[\frac{E^{2}\left(1+D^{2}+E^{2}\right)}{\left(1+E^{2}\right)\left(E^{2}+D^{2}\right)}\right]^{E^{2}}\left[\frac{D^{2}\left(1+D^{2}+E^{2}\right)}{\left(1+D^{2}\right)\left(D^{2}+E^{2}\right)}\right]^{D^{2}}\right\}\right)
\end{gathered}
$$

Here, the two geometrical parameters are equal to 1 . The exact value is 0.200044 .

We used analytical solutions to verify the hemisphere method (both point-toarea and area-to-area formulations).

We utilized the Gauss quadrature formula to integrate the elementary view factors from the hemisphere onto the area of the square. Here we used 5 Gauss points in each direction ( 25 points in total). We made use of the norm $L_{l}$ to compute the relative error, i.e. the difference between the exact solution $\left(V F_{\text {analytical }}=0.200044\right)$ and the integrated hemisphere's view factor $V F_{\text {hemisphere }}$.

$$
\varepsilon=\frac{\left|V F_{\text {analytical }}-V F_{\text {hemisphere }}\right|}{V F_{\text {analytical }}}
$$

We performed this calculation for $n_{\text {lat }}$ and $n_{\text {lon }}$ varying from 20 to 100 (it is not useful to plot the results for lower resolutions, the calculated error will be too high). The results are plotted at figure 4(a).

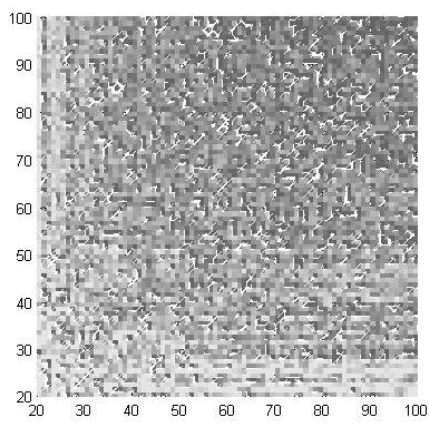

(a)
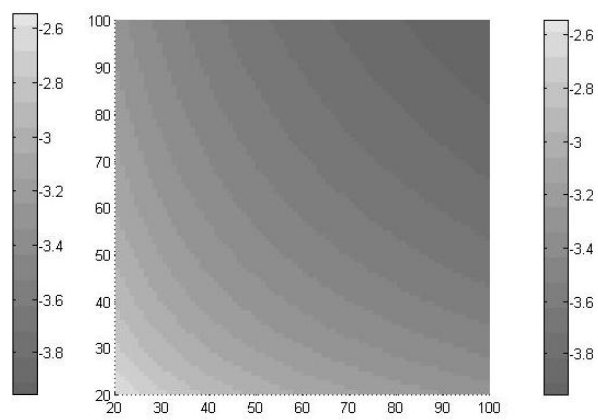

(b)

Figure 4: (a) Error for different resolutions. (b) Theoretical error for different resolutions. 
The abscissa $x$ and $y$ are the values of the parameters $n_{\text {lon }}$ and $n_{\text {lat }}$ respectively. The $z$ axis represents the logarithm of the relative error. In this figure, the error varies between -2.5 and -4 , i.e. $10^{-2.5}$ and $10^{-4}$.

We can observe that the error decreases when the resolution increases. The error can be approximated by $\varepsilon \approx \frac{1}{n_{\text {lon }} n_{\text {lat }}}$. This error is plotted at figure 4(b). For 10000 casted rays ( $n_{\text {lon }}=100$ and $n_{\text {lat }}=100$ ), the error will be around $10^{-4} 10^{-4}$.

Remark: during this test, we found some difficulties with this particular geometry. The vertical edges of the square are projected onto the hemisphere along meridians. When the mesh of the hemisphere and the projected edges are superimposed, nearly all the cells in the meridian are wrong. The error is independent of the colatitude resolution, around $\frac{1}{n_{l o n}}$. This is called discretization error. It gives oscillations in the error graphics. To prevent this case, we implemented a variant of the hemisphere, where the rays are randomly casted through each cell. We compute a mean value for each cell and we find an error close to $\frac{1}{n_{l o n} n_{l a t}}$. The mathematical theory which supports this method is not presented in this article but is developed in the internal report [17]. This random method has an evident drawback. The results are affected by the noise that characterizes all random processes. Nevertheless, this method is more reliable since it is not affected by the discretization error. If we consider the corresponding error plots, the oscillations disappear. Such error plots are shown in reference [17].

\section{Results}

In order to illustrate the hemisphere method, we implemented the well known scene called the Cornell box [1]. Picture 5 was obtained with a mesh of around 2000 triangular cells. We used the random version of the hemisphere, with 4 rays per cell. To display this view, we used the tone mapping method called mean value mapping [12] and linear interpolation.

The inter reflection of light is correctly modeled. The penumbra caused by the obstacles is also automatically computed.

Note that a colour copy of all these pictures is accessible from [18].

\section{Conclusions}

In this article, we have presented a simple method which allows us to compute the view factors from a point to surfaces of finite dimensions. This method allows us to keep a good physical comprehension of the problem. 


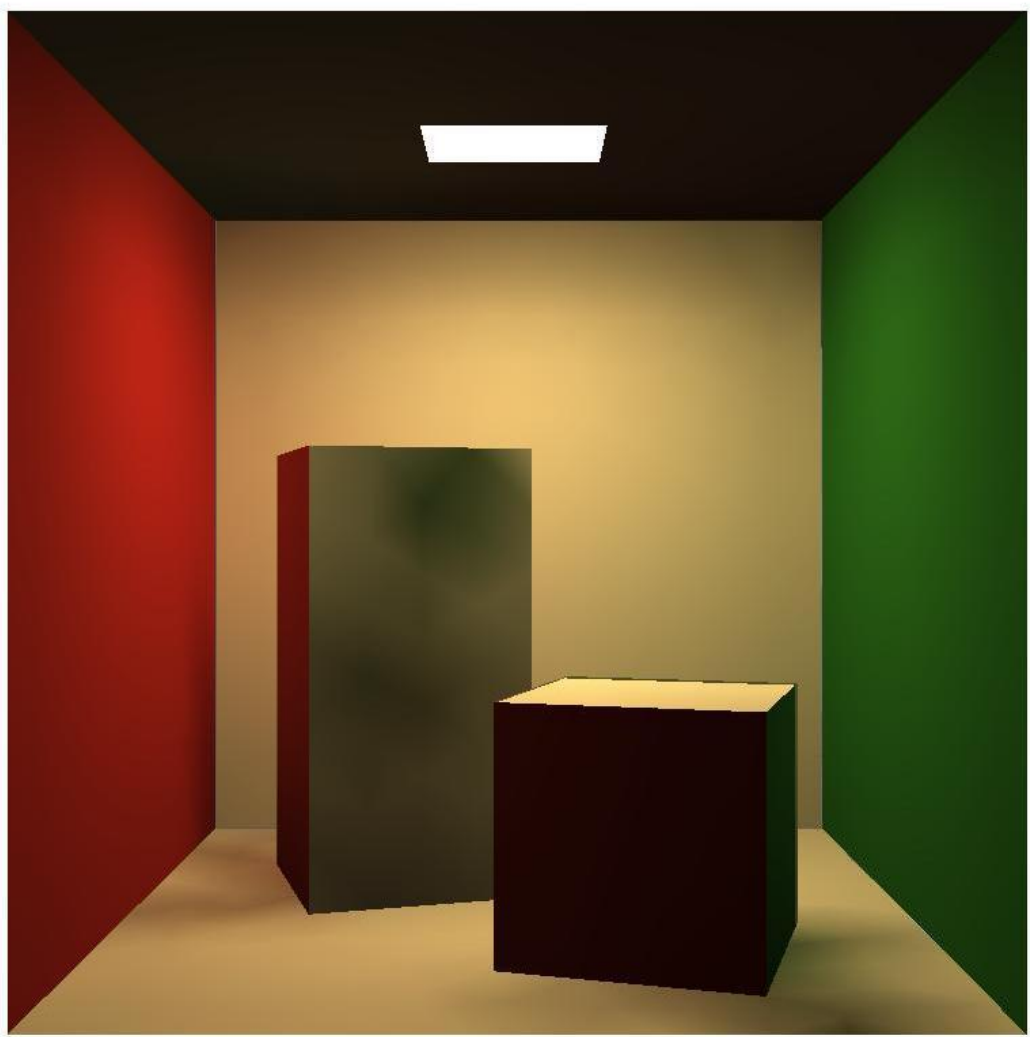

Figure 5: Cornell box using the hemisphere's method.

Compared to the hemi cube method, this method is faster and requires less memory. This method needs only one projection, the algorithm is simpler. All the elementary view factors are equal, which prevents useless over sampling and the storage of all the contributions to the view factors.

Compared to the single plane method, the hemisphere method is as fast. Nevertheless, we can study the whole hemisphere, without neglecting the surfaces close to the horizon.

\section{References}

[1] The Cornell Box, http://www.graphics.cornell.edu/online/box/.

[2] Donald P. Greenberg, Benneth Bataille, Cindy M. Goral, Kenneth E. Torrance, Modeling the interactions of light between diffuse surfaces. Computer Graphics, 6(2), pp. 213-222, 1984.

[3] W. R. Morgan, D. C. Hamilton, Radiant-interchange configuration factors, Technical report 2836, National Advisory Committee for Aeronautics, 1952. 
[4] Claude Puech, François Sillion, A general two-pass method integrating specular and diffuse reflection, Computer Graphics, 23(3), pp. 335-344, 1989.

[5] Claude Puech, François Sillion. Radiosity and Global Illumination, Morgan Kaufmann Publishers, Inc., 1994.

[6] John R. Howell. A Catalog of Radiation Heat Transfer Configuration Factors, McGraw Hill, 1982. http://www.me.utexas.edu/ howell/.

[7] H. Y. Wong. Handbook of Essential Formulae and Data on Heat Transfer for Engineers. Longman, 1976.

[8] Stéphane Jardon, Première approche sur l'étude des facteurs de vue, 2003, Mechanical Department, University of Liège, Belgium.

[9] James T. Kajiya, The rendering equation, SIGGRAPH, 20(4), 1986.

[10] T. G. Gonda, K. R. Johnson, A. R. Curran. Development of a signature supercode, Technical Proceedings 1938: Advances in Sensors, Radiometric Calibration, and Processing of Remotely Sensed Data (SPIE International Symposium on Optical Engineering and Photonics in Aerospace and Remote Sensing): Orlando, FL, 1993.

[11] Roberto Vivo, Maria José Vicent, Javier Lluch. Un método para el calculo del factor de forma alternativo al hemi-cubo. Universidad Politécnica de Valencia.

[12] Kresimir Matkovic. Tone mapping techniques and color image difference in global illumination. Master's thesis, Technischen Universität Wien, February 1998.

[13] Donald P. Greenberg, Michael F. Cohen, The hemi cube - a radiosity solution for complex environments, Computer Graphics, 19(3), pp. 31-40, 1985.

[14] John R. Wallace, Michael F. Cohen. Radiosity and Realistic Image Synthesis, Morgan Kaufmann Publishers, Inc, 1993.

[15] W. Nusselt, Graphische Bestimmung des Winkelverhaltnisses bei der Wärmestrahlung, Zeitschrift des Vereines Deutscher Ingenieure, 72(20), pp. $673,1928$.

[16] J. Lluch, R. Molla, P. Jorquera, R. Vivo, M. J. Vicent, Study of the form factor calculation by single polar plane. Department of Computer Systems and Computation, Universidad Politécnica de Valencia.

[17] P. E. Vueghs, Méthode de l'hémisphère - validation de la méthode, technical report, University of Liège, Belgium, 2005.

[18] LTAS - Infographie, Université de Liège, http://www.ulg.ac.be/ltas-cao/. 